Veronica Marie M. Mendoza, MD'

January E. Gelera, MD'

Christen-Zen I. Sison, MD'

Francis Aaron D. Dizon, MD²

Juan Miguel L. Manalo, MD²

'Department of Otorhinolaryngology

Head and Neck Surgery

University of Santo Tomas Hospital

${ }^{2}$ Department of Radiology

University of Santo Tomas Hospital

Correspondence: Dr. January E. Gelera Department of Otorhinolaryngology Head and Neck Surgery

University of Santo Tomas Hospital

España Blvd., Sampaloc, Manila 1015

Philippines

Phone: (632) 87313001 local 2411

Email:vignettejan@gmail.com

The authors declared that this represents original material that is not being considered for publication or has not been published or accepted for publication elsewhere, in full or in part, in print or electronic media; that the manuscript has been read and approved by all the authors, that the requirements for authorship have been met by each author, and that each author believes that the manuscript represents honest work.

Disclosures: The authors signed disclosures that there are no financial or other (including personal) relationships, intellectual passion, political or religious beliefs, and institutional affiliations that might lead to a conflict of interest.

Presented at the Philippine Academy of Rhinology Antonio L. Roxas International Research Contest, December 1, 2017. Manila Hotel, One Rizal Park, Manila.

\section{Radiologic Study of the Nasal Septal Swell Body and its Relationship to Septal Deviation}

\begin{abstract}
Objective: To compare the radiographic features of the nasal septal swell body (NSB) with the laterality of nasal septal deviation and investigate whether there is a correlation between the severity of the septal deviation and difference in NSB size.
\end{abstract}

\section{Methods:}
Design:
Retrospective Observational Study
Setting: Tertiary Private University Hospital
Participants: 30 paranasal sinus computerized tomography scans from January to

October 2017

Results: A septal deviation was present in $60 \%$ of the subjects. In $78 \%$ of cases with septal deviation, the NSB was noted to be significantly larger on the side opposite the nasal septal deviation $(p<.05)$.

Conclusion: The correlation between the severity of the septal deviation and difference in NSB size had a value of $(r=0.37)$ therefore, no positive correlation was established. Subjects with almost symmetric NSB measurements tend to have no septal deviation. On the other hand, the NSB is more prominent contralateral to a septal deviation.

Keywords: nasal septal swell body; septal deviation; inferior turbinate hypertrophy

The nasal septal swell body (NSB) is a distinct and widened region of the anterior nasal septum composed of septal cartilage, bone and a thick mucosal lining. ${ }^{1,2}$ This structure is located superior to the inferior turbinate and anterior to the middle turbinate and can be identified on anterior rhinoscopy, nasal endoscopy and on sinonasal imaging studies..$^{2-4}$ The NSB has been said to have similar characteristics and physiologic properties to the inferior turbinate (IT)..$^{2-6}$ It contains vasoactive tissue that may behave in a manner similar to that of the inferior turbinate, hence the term, septal turbinate..$^{2-7}$

The relationship of inferior turbinate hypertrophy and septal deviation has been discussed in the literature wherein inferior turbinate hypertrophy occurs compensatory to the presence of nasal septal deviation. ${ }^{1,3}$ Both the nasal septum and inferior turbinate have been studied well with regards to its function in nasal airflow regulation. ${ }^{3}$ However, the NSB receives little attention in the clinical setting and can be confused with a high septal deviation. ${ }^{4,5}$

In order to explore a possible relationship between the nasal septal body and nasal septal deviation (and whether there is a correlation between the severity of the septal deviation and 
difference in NSB size), this study aims to compare the radiographic features of the nasal septal swell body with the laterality of nasal septal deviation using paranasal sinus $\mathrm{CT}$ scans.

\section{METHODS}

With institutional review board approval (IRB-2017-10-194-TR), this retrospective study serially selected 30 paranasal sinus computerized tomography (CT) scans of patients aged 18 to 80 years old from the radiology department database of our hospital between the months of January and October 2017. The scans had been obtained using a Siemens Somatom Sensation 64 slices (Siemens Healthcare $\mathrm{GmbH}_{\text {, }}$ Erlangen, Germany). Coronal and axial images with a maximum of 1.0-millimeter thickness were included. Excluded from the study were $\mathrm{CT}$ scan images with evidence of nasal bone or nasal septum fracture and records of those with previous surgical intervention to the nasomaxillary area. The sample size of 30 was computed using the Fleiss et al. and Kelsey formula., ${ }^{8,9}$

The NSB was identified and the epicenter (the area of the greatest width) was marked as reference. The width of the septal body was separately measured from the widest lateral aspect of the nasal septal swell body up to the nasal septal cartilage on each side.' (Figure 1) The difference in septal body size on each side was measured and recorded.

The presence of septal deviation was also obtained from these images. The presence, laterality and degree of septal deviation were recorded. ${ }^{3}$ Two lines at an angle were used to assess the degree of septal deviation. The first line was drawn between the crista galli and the premaxillary area. The second line was from the crista galli to the most prominent portion of the nasal septum. ${ }^{3}$ (Figure 2) The degree of septal deviation was classified as: mild $\left(\leq 8^{\circ}\right)$, moderate $\left(9-15^{\circ}\right)$ and severe $\left(\geq 16^{\circ}\right) .{ }^{10}$ ClearCanvas Workstation version 13.1 (Synaptive Medical, Toronto, Canada) was used to measure the width and angles. Data was collected and tabulated using Microsoft Excel for Mac version 16.7 (Microsoft Corp. Redmond, WA, USA).

Data analysis was performed using IBM SPSS Statistics for Windows Version 20.0 (IBM Corp., Armonk, New York). Student's T- test was used to correlate the mean difference in the size of the NSB and the degree of septal deviation. Simple regression analysis using a Linear Model was utilized to show the correlation between the degree of severity of septal deviation and NSB size difference. $A$ value of $P<.05$ was considered statistically significant.

\section{RESULTS}

A total of 30 patient records ( 15 males and 15 females) met inclusion and exclusion criteria. Ages ranged from 18 to 80 years old (mean age 38 years old). The average total width of the septal body measured by adding the width of the septal body on each side of the septal cartilage was $9.5 \mathrm{~mm}$ (SD 1.5). The degree of septal deviation GNM was classified

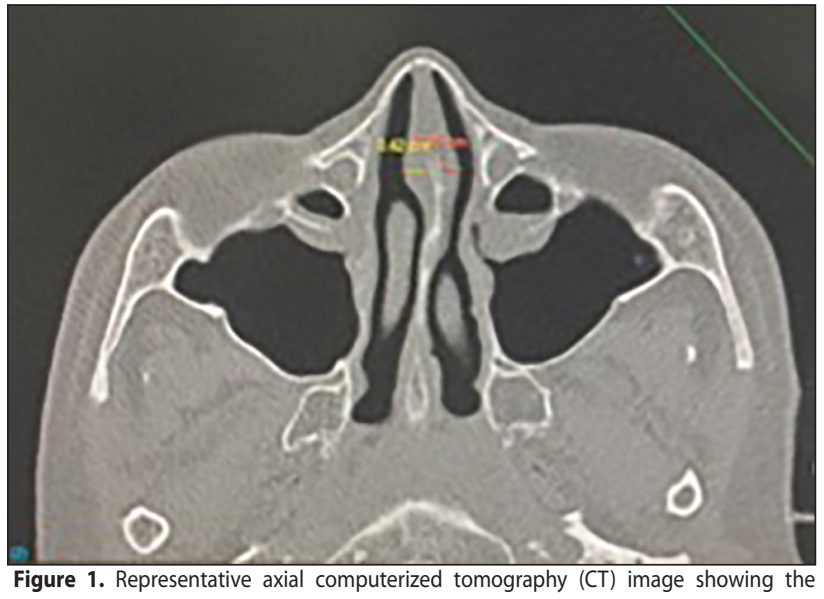
measurement of the nasal septal swell body on both sides.

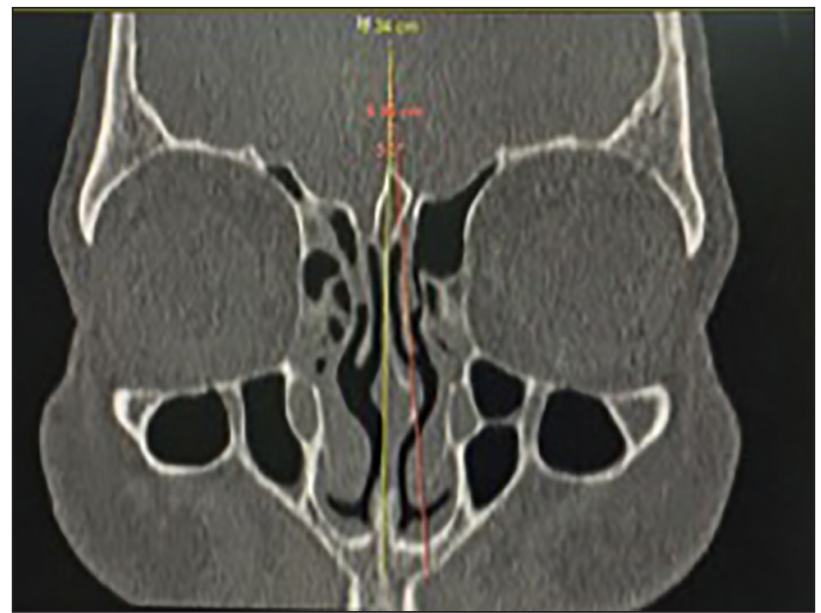

Figure 2. Representative coronal computerized tomography (CT) image showing the measurement of the degree of nasal septal deviation. The angle between the two lines was used to quantify the degree of septal deviation.

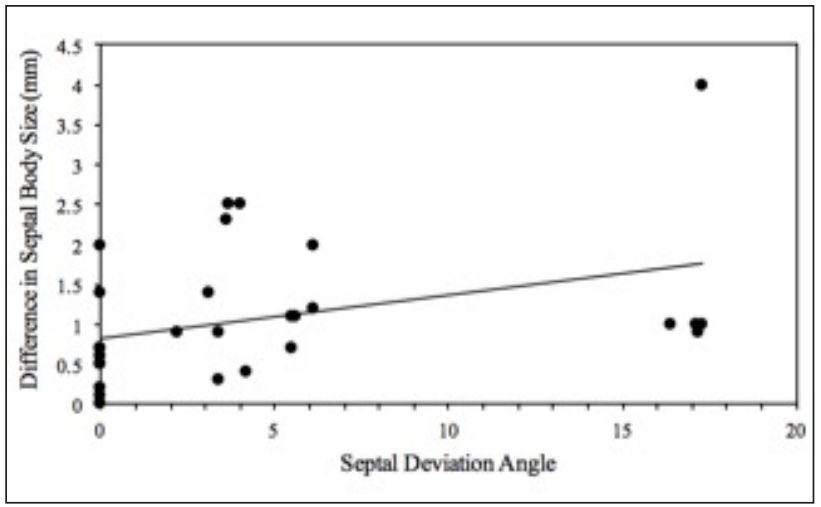

Figure 3. Scatter plot of difference in septal body size by degree of septal deviation. A linear regression model shows that there are inconclusive findings in the relationship between the degree of septal deviation and septal body size.

as mild $\left(\leq 8^{\circ}\right)$ in 13 cases and severe $\left(\geq 16^{\circ}\right)$ in 5 cases. No cases were classified as moderate $\left(9-15^{\circ}\right)$.

A distinct nasal septal swell body was identified in all 30 cases. The presence of septal deviation was noted in 18 out of the $30(60 \%)$. In 14 of the 18 with septal deviation (78\%), the septal body was noted to 


\section{ORIGINAL ARTICLES}

be significantly larger on the side opposite the nasal septal deviation $(p<.05)$. No significant asymmetry of the nasal septal swell body was identified in the 12 cases with absence of septal deviation. The mean difference in septal body size was $1.58 \mathrm{~mm}$ in cases with severe septal deviation and $0.98 \mathrm{~mm}$ in cases with mild septal deviation. There was no statistically significant difference found between the septal body size of patients with severe septal deviation and those with mild septal deviation $(p<.05)$.

The correlation between the severity of the septal deviation and difference in the septal body size on the two sides of the septum was not classified as high ( $r=0.37$ ). A positive $r$ value signifies that there is a positive relationship between the severity of septal deviation and difference in the septal body size. Figure 3 shows the result of a simple regression analysis using a linear model for which $R^{2}=0.138$ was obtained. This denotes that only $13.8 \%$ of the variance in the septal body size difference is due to the severity of septal deviation.

\section{DISCUSSION}

Nasal airflow is regulated predominantly by the nasal turbinates. On the other hand, the NSB has been said to have similar characteristics and properties as the inferior turbinate. ${ }^{1,11}$ It contains vasoactive tissue that may behave in a manner similar to the inferior turbinate wherein arteriovenous congestion would cause nasal airway obstruction., ${ }^{4,12}$ However, its role in nasal airflow regulation remains unclear. Several sources in the literature observe that patients with septal deviation are often found to have significant inferior turbinate hypertrophy on the side opposite the septal deviation as a counterbalance mechanism. ${ }^{13,14}$ This compensatory hypertrophy of the inferior turbinate protects the more spacious nasal side from crusting, drying, altered air filtration and mucociliary flow due to the excess air. ${ }^{13,14,15}$

The results of our study suggest that the NSB is more prominent contralateral to a septal deviation. These findings echo those seen with inferior turbinate hypertrophy ${ }^{11}$ and may corroborate those of Setlur and Goyal who concluded (based on the similarities of the NSB and inferior turbinate) that the NSB may have a role in regulating nasal airflow and contribute to nasal obstruction. ${ }^{3}$ Recent evidence has shown that direct decongestant application isolated to the NSB resulted in a reduction in nasal obstruction symptoms as well as improved scores on anterior rhinomanometry, acoustic rhinometry and peak nasal inspiratory flow. ${ }^{12}$

Unfortunately, there was no statistically significant correlation between the severity of the septal deviation and difference in the septal body size in our study, differing from the theoretical results obtained from previous studies. ${ }^{3,4,5}$ This may be due to the small sample size used for the study, and the subsequent number of subjects with septal deviation (18 out of 30 ).
Since this study focused mainly on radiographic characteristics of the NSB and septal deviation and not on its histologic composition, it is recommended that the vascular and glandular structures of the NSB be investigated further, in order to elucidate the physiologic properties of the NSB and how they might affect nasal airflow. Furthermore, the emphasis on the NSB as a distinct and separate structure may direct novel surgical procedures or medical therapies specific to its management in the future.

Our study showed that those with almost symmetric septal body measurements tend to have no septal deviation, while patients with asymmetric NSB hypertrophy tend to have septal deviation contralateral to the side with hypertrophy, but our results are not conclusive. Whether this relationship may be similar to that of septal deviation and compensatory contralateral inferior turbinate hypertrophy, and whether it can suggest that the NSB may have an impact on nasal airflow regulation similar to that of the inferior turbinate, needs further study.

\section{REFERENCES}

1. Costa DJ, Sanford T, Janney C, Cooper M, Sindwani R. Radiographic and anatomic characterization of the nasal septal swell body. Acrh Otolaryngol Head Neck Surg. 2010 Nov; 136(11): 1107-1110. DOI: 10.1001/archoto.2010.201; PMID: 21079165.

2. Lapeña JF. Tuberculum Septi. Brunei Int Med J. 2011 Aug; 7(4): 239.

3. Setlur J, Goyal P. Relationship between septal body size and septal deviation. Am J Rhinol Allergy. 2011 Nov-Dec; 25(6): 397-400. DOI: 10.2500/ajra.2011.25.3671; PMID: 22185743.

4. Elwany S, Salam SA, Soliman A, Medanni A, Talaat E. The septal body revisited. J Laryngol Otol. 2009 Mar; 123(3):303-308. DOI: 10.1017/S0022215108003526; PMID: 18796179.

5. Wotman M, Kacker A. Should otolaryngologists pay more attention to nasal swell bodies? Laryngoscope. 2015 Aug; 125(8):1759-1760. DOI: 10.1002/lary.25144; PMID: 25600033.

6. Arslan M, Muderris T, Muderris S. Radiological study of the intumescentia septi nasi anterior. $J$ Laryngol Otol. 2004 Mar; 118(3): 119-201. DOI: 10.1258/002221504322927964; PMID: 15068516.

7. Wexler D, Braverman I, Amar M. Histology of the nasal septal swell body (septal turbinate). Otolaryngol Head Neck Surg. 2006 Apr; 134(4): 596-600. DOI: 10.1016/j.otohns.2005.10.058; PMID: 16564379.

8. Kelsey JL, Whittemore AS, Evans AS, Thompson WD. Methods in Observational Epidemiology. Oxford: Oxford University Press; 1996.

9. Fleiss JL. Statistical Methods for Rates and Proportions. Hoboken, New Jersey: John Wiley \& Sons; 1981.

10. Elahi MM, Frenkiel S, Fageeh N. Paraseptal structural changes and chronic sinus disease in relation to the deviated septum. J Otolaryngol. 1997 Aug; 26(4): 236-240. PMID: 9263892.

11. San T, Muluk NB, Saylisoy S, Acar M, Cingi C. Nasal septal body and inferior turbinate sizes differ in subjects grouped by sex and age. Rhinology. 2014 Sep; 52(3): 231-237. DOI: 10.4193/ Rhin13.138; PMID: 25271528

12. Wong E, Deboever N, Chong J, Sritharan N, Singh N. Isolated topical decongestion of the nasal septum and swell body is effective in improving nasal airflow. Am J Rhinol Allergy. 2020 February. DOI: 10.1177/1945892420902913.

13. Akoglu E, Karazincir S, Balci A, Okuyucu S, Sumbas H, Dagli AS. Evaluation of the turbinate hypertrophy by computed tomography in patients with deviated nasal septum. Otolaryngol Head Neck Surg. 2007 Mar; 136(3):380-384. DOI: 10.1016/j.otohns.2006.09.006; PMID: 17321863.

14. Egeli E, Demerici L, Yazycy B, Harputluoglu U. Evaluation of the inferior turbinate in patients with deviated nasal septum by using computed tomography. Laryngoscope. 2004 Jan; 114(1):113-117. DOI: 10.1097/00005537-200401000-00020; PMID: 14710005.

15. Estomba CC, Schmitz TR, Echeverri CO, Reinoso FA, Velasquez AO, Hidalgo CS. Compensatory hypertrophy of the contralateral inferior turbinate in patients with unilateral nasal septal deviation. Otolaryngol Pol. 2015 April; 69 (2): 14-20. DOI: 10.5604/00306657.1149568. 